\title{
ARTICLE
}

\section{Relationship between gene expression and the accumulation of catechin during spring and autumn in tea plants} (Camellia sinensis L.)

\author{
Min Liu ${ }^{1}$, Heng-lu Tian ${ }^{1}$, Jian-Hua Wu ${ }^{2}$, Ren-Rong Cang ${ }^{3}$, Run-Xian Wang ${ }^{3}$, Xiao-Hua Qi ${ }^{1}$, Qiang Xu ${ }^{1}$ and Xue-Hao Chen ${ }^{1}$
}

The tea plant (Camellia sinensis L.) is an important commercial crop with remarkably high catechin concentrations. Tea is popular worldwide given the plant's health benefits. Catechins are the main astringent substance in tea and are synthesized mainly via the phenylpropanoid pathway. In this study, eight cultivars of tea plants harvested both in spring and autumn were used to investigate differences in catechin concentrations by using high-performance liquid chromatography. The expression levels of genes associated with catechin biosynthesis were investigated using reverse transcription-quantitative polymerase chain reaction. The results indicated that the total catechin (TC) concentrations were significantly higher in tea plants harvested in autumn than in those harvested in spring, based on higher concentrations of epigallocatechin (EGC) in autumn tea $(P<0.01)$. The expression of the genes phenylalanine ammonia-lyase $(P A L)$, flavanone 3-hydroxylase $(F 3 H)$, flavonoid $3^{\prime}, 5^{\prime}$-hydroxylase $\left(F 3^{\prime} 5^{\prime} H\right)$, dihydroflavonol 4-reductase $(D F R)$, and anthocyanidin synthase (ANS) is closely related to the TC content of tea plants in both spring and autumn. Positive correlations between $P A L$, cinnamate 4-hydroxylase $(C 4 H), F 3 H$, and DFR expression and EGC accumulation in autumn tea were identified, with correlation coefficients of $0.710,0.763,0.884$, and 0.707 , respectively. A negative correlation between ANS expression level and EGC concentrations in tea plants harvested in spring was noted ( $r=-0.732$ ). Additionally, negative correlations between F3H and ANS expression levels and the catechin content were identified in spring tea, whereas the correlations were positive in autumn tea. Significant differences in the $\mathrm{F} 3 \mathrm{H}$ and $\mathrm{ANS}$ expression levels between spring and autumn tea indicate that $\mathrm{F} 3 \mathrm{H}$ and $\mathrm{ANS}$ are potentially key genes affecting catechin accumulation in tea plants.

Horticulture Research (2015) 2, 15011; doi:10.1038/hortres.2015.11; Published online: 1 April 2015

\section{INTRODUCTION}

Tea is a globally economically important commodity and is enjoyed by people worldwide because of its health benefits. ${ }^{1}$ Catechins are the primary astringent substances in tea. Catechins have multiple effects on human health and play important antibacterial, antiviral, anti-radiation, and anti-aging roles. In addition, these compounds are involved in the prevention of cardiovascular disease and cancer. $^{2-6}$ They are abundant in the young leaves and buds of tea plants and can be divided into non-esterified catechins (including catechin (C), epicatechin (EC), gallocatechin (GC), epigallocatechin $(E G C)$ ), and esterified catechins (including epicatechin gallate (ECG) and epigallocatechin gallate (EGCG)). ${ }^{7}$

Catechins account for approximately $70 \%$ of all polyphenols in tea and are derived from multiple branches of the phenylpropanoid biosynthetic pathway, one of the most characterized secondary metabolic routes in plant systems. ${ }^{8-10}$ Flavan-3-ols (also known as catechins) are mainly produced via the naringenin-chalcone $\rightarrow$ naringenin $\rightarrow$ dihydrokaempferol pathway (Ashihara et al., 2010). ${ }^{11}$ As shown in Figure 1, the steps to produce dihydromyricetin are catalyzed by the following enzymes: phenylalanine ammonia-lyase (PAL), cinnamate 4-hydroxylase $(\mathrm{C} 4 \mathrm{H})$, chalcone synthase $(\mathrm{CHS})$, chalcone isomerase $(\mathrm{CH})$, flavanone 3-hydroxylase $(\mathrm{F} 3 \mathrm{H})$, flavonoid 3'-hydroxylase $\left(F 3^{\prime} H\right)$, and flavonoid $3^{\prime}, 5^{\prime}$-hydroxylase $\left(F 3^{\prime} 5^{\prime} H\right) .^{12-18}$ Dihydroflavonol 4-reductase $(D F R)$ catalyzes important steps in the control of metabolic fluxes, which feed into biosynthetic pathway branches, leading to the production of anthocyanins and proanthocyanidins. ${ }^{19}$ Non-esterified catechins are produced in steps involving sequential reactions catalyzed by leucoanthocyanidin 4-reductase (LAR), anthocyanidin synthase (ANS), and anthocyanidin reductase (ANR). ${ }^{20-22}$ EGC and EC are converted into esterified catechins (EGCG and ECG) via the sequential action of flavan-3-ol gallate synthase (FGS), ${ }^{11}$ and various catechin monomers are synthesized from dihydrogen arbutus pigment. $^{10,11,23}$

To date, studies on catechin biosynthesis have mainly focused on the influence of light, ${ }^{24,25}$ drought, ${ }^{26}$ high ultraviolet (UV) radiation levels, ${ }^{27}$ low temperature, ${ }^{28}$ and pathogen infection, ${ }^{29}$ as well as on the different maturing tissues, ${ }^{30}$ the albino phenomenon, ${ }^{31}$ and dark treatment. ${ }^{32}$ In general, the quality of sweetness, flavor, texture, and taste in spring tea is better than that in autumn tea. ${ }^{33}$ However, most previous studies have focused on the effects of environmental factors and alterations in amino acids, polyphenols, and caffeine content during seasonal changes in tea plants. ${ }^{34,35}$ Chemical analysis of bud/leaves harvested in spring revealed a higher concentration of amino acids and aroma characteristics, but lower levels of total polyphenols and caffeine than those in tea plant leaves harvested in autumn. ${ }^{34,35}$ Little information is available on the molecular biology of catechin accumulation and its biosynthesis-related gene expression in tea plants during the spring and autumn seasons.

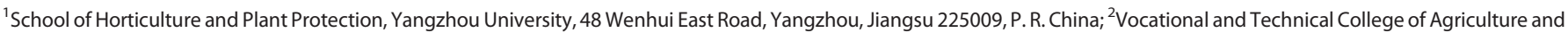
Forestry (School of Agriculture), 18 Wenchang East Road, Jurong, Jiangsu 212400, P. R. China and ${ }^{3}$ Tea Research Institute, Jurong, Jiangsu 212400, P. R. China

Correspondence: Xue-Hao Chen (xhchen@yzu.edu.cn)

Received: 10 November 2014; Revised: 23 February 2015; Accepted: 24 February 2015
} 


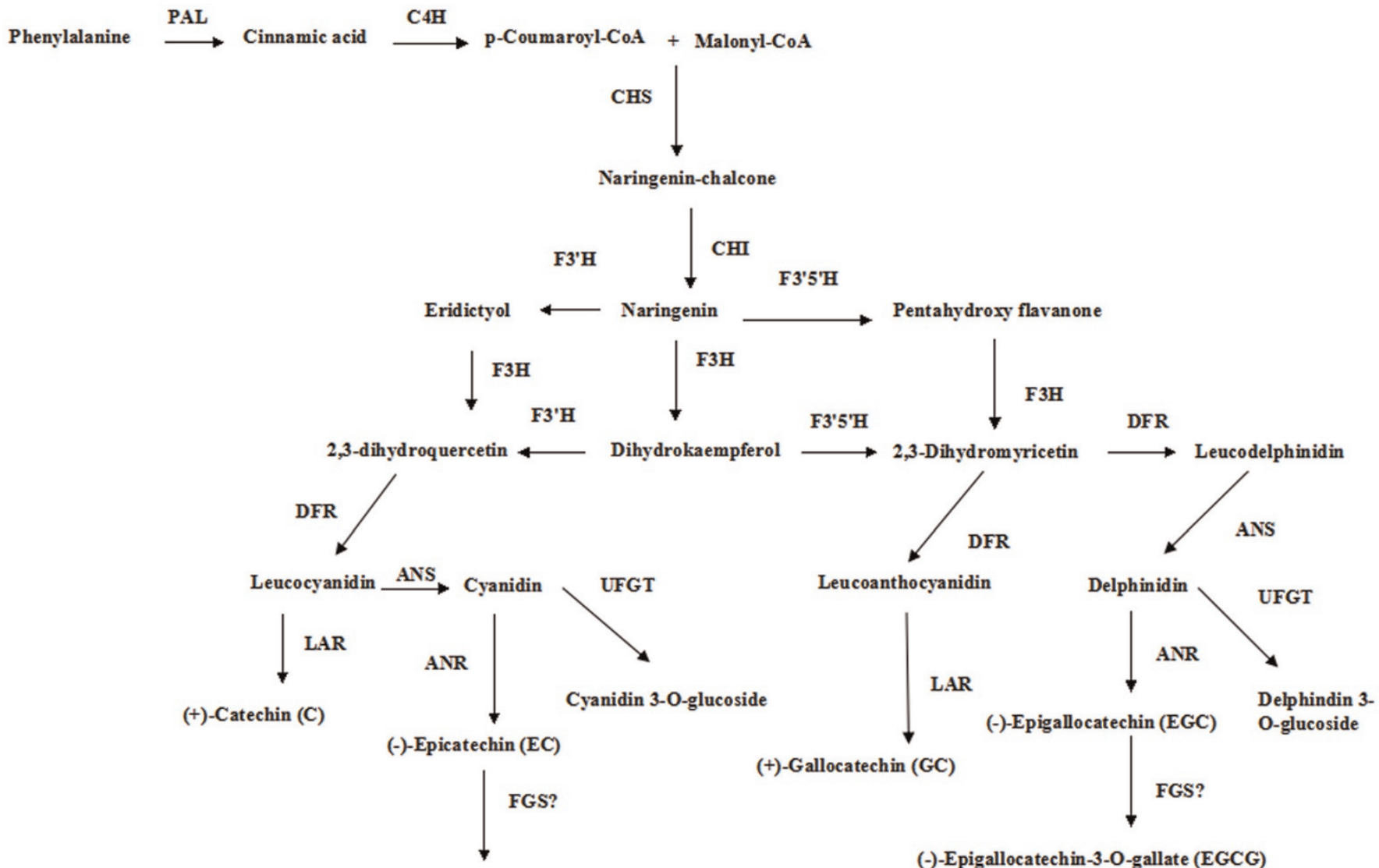

(-)-Epicatechin-3-O-gallate (ECG)

(-)-Epigallocatechin-3-0-gallate (EGCG)

Figure 1. Possible biosynthetic pathways of catechins in tea (Camellia sinensis) leaves. ${ }^{9,10,22}$ Key enzyme names are: PAL, phenylalanine ammonia-lyase; $\mathrm{C4H}$, cinnamate 4-hydroxylase; $\mathrm{CHS}$, chalcone synthase; $\mathrm{CHI}$, chalcone isomerase; $\mathrm{F3H}$, flavanone 3-hydroxylase; $\mathrm{F3}$ ' $\mathrm{H}$, flavonoid 3'-hydroxylase; F3' 5' H, flavonoid 3',5'-hydroxylase; DFR, dihydroflavonol 4-Reductase; LAR, leucocyanidin reductase; ANR, anthocyanidin reductase; ANS, anthocyanidin synthase; FGS, flavan-3-ol gallate synthase.

In this study, we analyzed the catechin content and expression levels of genes involved in the biosynthesis of catechins in eight tea plant cultivars harvested both in spring and autumn (hereafter spring and autumn tea, respectively). These results provide insight into the possible mechanisms regulating catechin biosynthesis in tea plants and may lead to a better understanding of differences in catechin content between spring and autumn tea and the underlying genetic mechanisms.

\section{MATERIALS AND METHODS}

Plant samples

Fresh tea bud leaves consisting of the apical bud and the leaf closest to the apex were harvested from the Tea Research Institute, Jiangsu Province, P. R. China, between 09:00 am and 12:00 am on 2 April and 13 September 2013 from eight tea cultivars (Camellia sinensis), including 'Longjingchangye' tea (LJCY), 'Anjibaicha' tea (AJBC), 'Fuding-dahao' tea (FDDH), 'Fuding-dabai' tea (FDDB), 'Zhenong117' tea (ZN117), 'Wuniuzao' tea (WNZ), 'Pingyang-tezao' tea (PYTZ), and 'Longjing43' tea (LJ43). The samples were divided into two: half of each sample was frozen immediately in liquid nitrogen and stored at $-80{ }^{\circ} \mathrm{C}$ until RNA extraction. The other half was microwaved (AX-1500Y(R); Sharp, Osaka, Japan) at high power $800 \mathrm{~W}$ for 2 min to terminate all polyphenol oxidase activity ${ }^{36}$ and maintained at $-80{ }^{\circ} \mathrm{C}$ until catechin extraction. All experiments included tests on three separate biological replicates.

\section{Catechin concentrations}

Catechin extraction was performed using $0.2 \mathrm{~g}$ of fresh tea bud leaves from each sample. ${ }^{37}$ Catechins were analyzed according to Zhang et al. ${ }^{38}$ with some modifications. Bud leaf tissue $(0.2 \mathrm{~g} \mathrm{FW})$ was homogenized with a mortar and pestle and placed in a 10-ml centrifuge tube containing $6 \mathrm{ml}$ of $70 \%(\mathrm{v} / \mathrm{v})$ methanol. The tube was heated at $80{ }^{\circ} \mathrm{C}$ for $20 \mathrm{~min}$ in a shaking thermal water bath and then centrifuged (Centrifuge 5810 R; Eppendorf, Hamburg, Germany) at $4{ }^{\circ} \mathrm{C}$ and $10000 \mathrm{rpm}$ for $15 \mathrm{~min}$. The supernatant was transferred into a new $10-\mathrm{ml}$ centrifuge tube and filtered through a $0.45-$ $\mu \mathrm{m}$ organic membrane; $70 \%(\mathrm{v} / \mathrm{v})$ methanol was added to dilute the supernatant to $100 \mathrm{ml}$. A standard curve was prepared by weighing standard solutions of C, GC, EGC, EC, EGCG, and ECG purchased from Sigma-Aldrich (Sigma, Milwaukee, WI, USA) in $70 \%(\mathrm{v} / \mathrm{v})$ methanol. ${ }^{37}$ As a result, stock solutions containing all six catechins at $100 \mathrm{mg} \mathrm{ml}^{-1}, 50 \mathrm{mg} \mathrm{ml}^{-1}, 25 \mathrm{mg}$ $\mathrm{ml}^{-1}, 12.5 \mathrm{mg} \mathrm{ml}^{-1}$, and $6.25 \mathrm{mg} \mathrm{ml}^{-1}$ were prepared. Different concentrations of standard solution were prepared via dilution of the stock using the same solvent, $70 \%(\mathrm{v} / \mathrm{v})$ methanol. Extracted samples and standards were stored at $4{ }^{\circ} \mathrm{C}$ and protected from light before measurement.

High-performance liquid chromatography (HPLC) analysis was performed in an L-2000 HPLC-UV detector (Hitachi, Tokyo, Japan) with an injection volume of $10 \mathrm{ml}$ and a $\mathrm{C}_{18}$ ODS column $(250 \mathrm{~mm} \times 4.6 \mathrm{~mm}, 5 \mu \mathrm{m}$; Phenomenex, Tokyo, Japan) plus a $\mathrm{C}_{18}$ guard column $(10 \mathrm{~mm} \times 3.0 \mathrm{~mm})$ at an oven temperature of $30{ }^{\circ} \mathrm{C}$. Mobile phase A consisted of a 5:95 (v/v) mix of acetonitrile:double distilled water containing $0.05 \%(\mathrm{v} / \mathrm{v})$ orthophosphoric acid; mobile phase $B$ consisted of a 50:50 (v/v) mix of acetonitrile:double distilled water containing $0.05 \%(\mathrm{v} / \mathrm{v})$ orthophosphoric acid. The flow rate was $0.5 \mathrm{ml}$ $\mathrm{min}^{-1}$, and the detecting wavelength was $231 \mathrm{~nm}$. The gradient elution procedure is presented in Table $1 .^{38}$ Peaks were identified by comparing sample retention times to those of authentic standards.

RNA extraction and quantitative RT-PCR

Total RNA from bud leaves was isolated using TRlzol reagent (Invitrogen, Carlsbad, CA, USA, http://www.invitrogen.com) according to the manufacturer's instructions. First-strand cDNAs were synthesized using the FastQuant 
Table 1. Gradient elution profile for HPLC analysis

\begin{tabular}{lrlllrr}
\hline \multirow{2}{*}{$\begin{array}{l}\text { Mobile phase } \\
(\%(\mathrm{v} / \mathrm{v}))\end{array}$} & 0 & 10 & 20 & 30 & 31 & 35 \\
\cline { 2 - 7 } & 9 & 10 & 15 & 20 & 9 & 9 \\
$\mathrm{~A}$ & 91 & 90 & 85 & 80 & 91 & 91 \\
\hline
\end{tabular}

Compositions of mobile phase A: 5:95 (v/v) mix of acetonitrile:double distilled water containing 0.05\% (v/v) orthophosphoric acid and mobile phase B: a 50:50 (v/v) mix of acetonitrile:double distilled water containing $0.05 \%(\mathrm{v} / \mathrm{v})$ orthophosphoric acid. The elution method was based on the method of Zhang et al. ${ }^{38}$

RT kit (with gDNase) (TIANGEN, Beijing, China, http://www.tiangen.com). According to the TIANGEN SuperReal PreMix Plus (SYBR Green) kits (TIANGEN, Beijing, China, http://www.tiangen.com), quantitative real-time (qRT) PCR was performed on a MiniOpticon Real-Time PCR system (Bio-Rad Laboratories Inc., USA). All primers used for qRT-PCR are listed in Table 2. After completion of the reactions, the threshold cycle $\left(C_{T}\right)$ value for each reaction was recorded, and the fold difference in transcript level between greenhouse and open field samples was calculated. The mean values of three replicates and relative to a $\beta$-actin standard are given.

Statistical analysis of the data

The data are expressed as the mean \pm standard deviation (SD) for three replicates. Relative quantification values were calculated using the $2^{-\Delta \Delta C}$ method. ${ }^{39}$ The data were subjected to analysis of variance (ANOVA), and the statistical significance of differences between groups was assessed via Student's $t$-test using SPSS (Statistical Package for the Social Sciences) 16.0 statistical software (SPSS Inc., Chicago, IL, USA). For multiple variable comparisons, data were analyzed by two-way ANOVA followed by Tukey's test. The correlation was analyzed via Pearson correlation. The $P$-values between catechins and gene expression were analyzed by pair comparisons between eight cultivated varieties. $P$-values $<0.05$ were considered significant.

\section{RESULTS}

Differences in catechin concentrations between spring and autumn teas

Six characteristic tea catechins were successfully extracted from bud leaves (Figure 2). The most abundant catechins included the esterified catechins EGCG and ECG, which accounted for approximately $60 \%$ of the total catechin (TC), and only low amounts of nonesterified catechins were noted in spring tea (Table 3). EGCG was the most abundant catechin in tea plants harvested in spring and autumn, and EC was the least abundant (Figure 3). These results are consistent with a previous report indicating that EGCG is generally the most abundant tea catechin, whereas $C$ and $E C$ concentrations are rather low. ${ }^{40}$ Tea plants harvested in autumn had higher EGC concentrations, followed by EGCG, C, and GC, but lower EC and ECG concentrations than in spring tea (Figure 3). With the higher EGC concentrations, esterified catechins, including EGCG and ECG, only accounted for approximately $50 \%$ of the TC of tea plants harvested in autumn, which was $10 \%$ lower than that in spring tea (Table 3 ).

To explore the effects of cultivar, season, and cultivar $\times$ season on catechin concentrations in tea plants, data were analyzed by two-way ANOVA followed by Tukey's test. As shown in Table 4, significant differences in catechin concentrations were identified in eight tea plant cultivars and two seasons (spring and autumn). A significant difference in cultivar $\times$ season was also noted. These results indicate that different cultivars and seasons affect catechin accumulation in tea plants.

\section{Expression of relevant genes}

The expression patterns of flavonoids (including catechin) and genes that are involved in the biosynthesis of catechins, such as $P A L, C 4 H$, CHS, CHI, F3H, F3' H, F3' $5^{\prime} H$, DFR, LAR, ANS, and ANR, were examined. The relative gene expression in spring tea and the correlation coefficients $(r)$ are listed in Table 5. ANS expression exhibited a significantly negative correlation with EGC accumulation, but $F 3^{\prime} H$ and $D F R$ expression were significantly positively correlated with EGCG accumulation. The relative expression levels of $P A L, D F R$, and $L A R$ in tea plants harvested in spring were significantly positively correlated with increased TC, whereas $F 3 H, F 3^{\prime} 5^{\prime} H$, and ANS gene expression were negatively correlated with increased TC (Table 5).

As shown in Table 6, EGC concentrations were closely associated with $P A L, C 4 H, F 3 H$, and DFR expression in autumn tea. A significantly positive correlation between $P A L$ and DFR expression and EGCG accumulation was noted. PAL, C4H, F3H, DFR, and ANS expression levels in tea harvested in autumn were significantly positively correlated with increased TC. In contrast, $F^{\prime}{ }^{\prime} 5^{\prime} H$ expression was negatively correlated with increased TC.

From Tables 5 and 6, correlations between catechin content and $P A L, F 3 H, F^{\prime} 5^{\prime} H, D F R$, and ANS gene expression were observed in the bud leaves of eight varieties in spring and autumn. A positive correlation between TC and $L A R$ expression $(r=0.707)$ was observed in spring tea, but a positive correlation was noted between $\mathrm{C} 4 \mathrm{H}$ expression and TC levels in autumn tea. F3H and ANS expression were downregulated in autumn tea as compared with spring tea, and catechin accumulation was higher in autumn tea than in spring tea (Figure 4). Catechin concentrations were negatively correlated with $\mathrm{F} 3 \mathrm{H}$ and $\mathrm{ANS}$ expression levels in spring tea but positively correlated in autumn tea (Tables 5 and 6).

\section{DISCUSSION}

Differences in catechin concentrations between spring and autumn tea

Catechins are the main astringent substances in tea and are important components of tea quality. Of the various catechins, ECs (including EC

Table 2. Primer sequences used for reverse transcription-quantitative PCR

\begin{tabular}{|c|c|c|c|}
\hline Gene name & GenBank Accession No. & Primer sequence $\left(5^{\prime} \rightarrow 3^{\prime}\right)$ & Length of product (bp) \\
\hline$P A L$ & D26596 & Forward: TCCTTGCCAATCCTGTAA Reverse: CAACTGCCTCGGCTGTCT & 105 \\
\hline DFR & AB018685 & Forward: ATGACTGGCTGGATGTATT Reverse: TGTTGGCATTATGAAAGG & 161 \\
\hline ANS & AY830416 & Forward: GACACCAACCGACTACATT Reverse: TGCCTCCAACTTCTTTCT & 134 \\
\hline$\angle A R$ & AY169406 & Forward: TGAAGTATGCAGCCTCTAC Reverse: CAGTGTTTCCATCCGTCT & 196 \\
\hline $\mathrm{F} 3 \mathrm{H}$ & AY641370 & Forward: TGGAGGGCTGTAACGGAG Reverse: ACTGTGGGCATTTCGGGTAG & 164 \\
\hline$F 3^{\prime} 5^{\prime} H$ & DQ184358 & Forward: TTGAGTTGTCGCCGTGAG Reverse: AAATAGCCTGCGGTGGTC & 167 \\
\hline $\mathrm{C} 4 \mathrm{H}$ & AY641731 & Forward: CTGGCTATGACATCCCTG Reverse: AACTCCTCCTTCCGACAC & 184 \\
\hline $\mathrm{CHI}$ & DQ904329 & Forward: CTTAGAGGCACTGACACC Reverse: CTTAATCGGAAAGGAGAC & 163 \\
\hline $\mathrm{CHS}$ & AY169403 & Forward: TGCCATTGACGGACACCT Reverse: GAATGCTTCCGCCAAACT & 128 \\
\hline$F 3^{\prime} H$ & GQ438849 & Forward: AAAGGGCTCAACTCTTCTT Reverse: AACTGGACCATACGCAAC & 210 \\
\hline ANR & AY641729 & Forward: TAAATGGGTTGAAAGGTATG Reverse: GCTCGGGAACACTGGTAT & 150 \\
\hline UFGT & GH618816 & Forward: CACCACTTCCGAATGACA Reverse: GCAGACCAGCCGTTTATC & 135 \\
\hline$\beta$-actin & FE861557 & Forward: TCGCATCCCTAAGCACCT Reverse: CACCGTCATCAACGCAAT & 175 \\
\hline
\end{tabular}


a

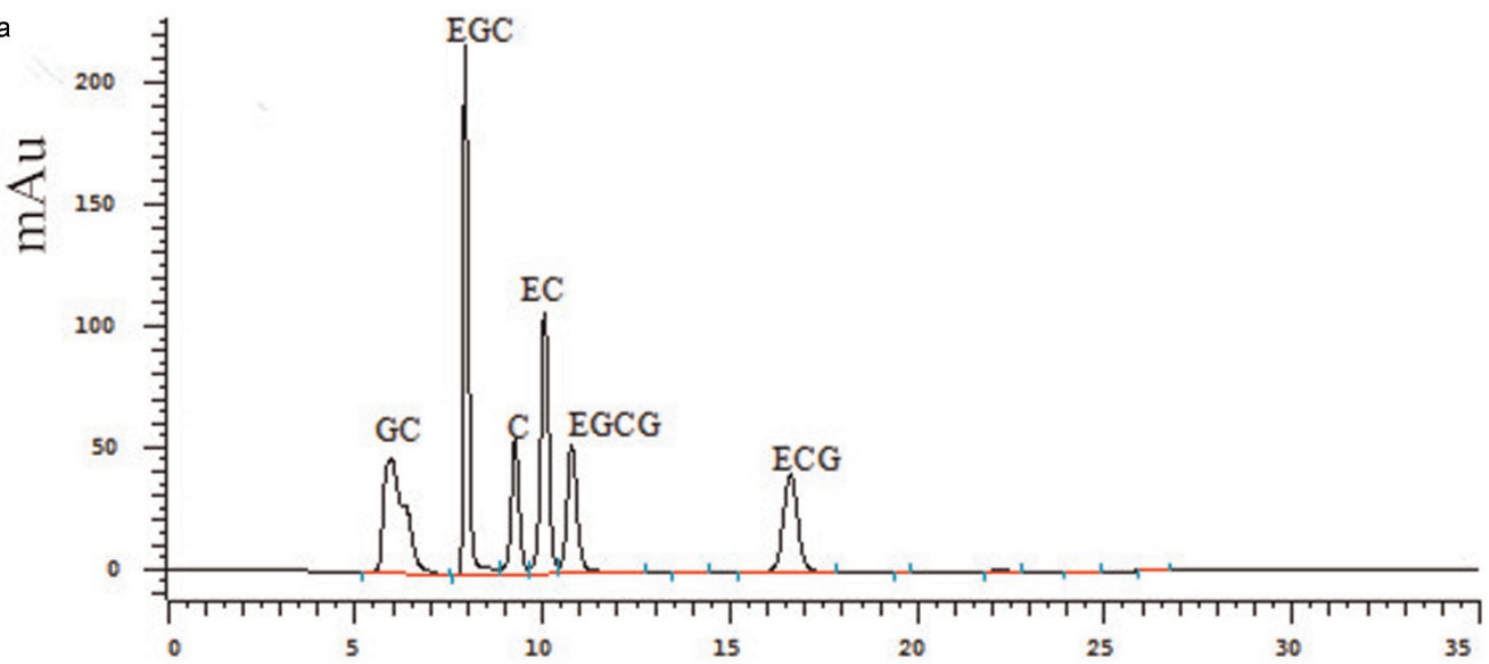

$b$

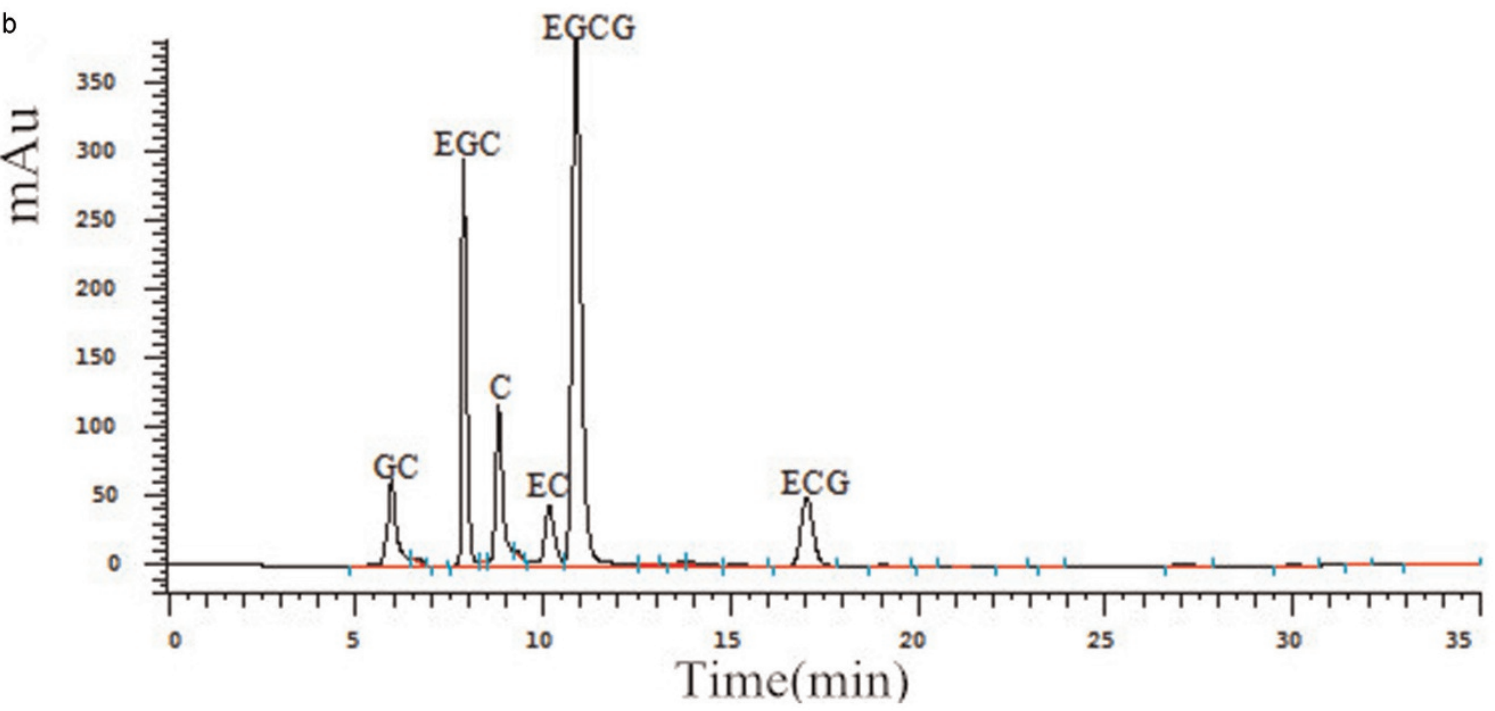

Figure 2. HPLC elution profiles of six catechin derivatives in tea bud leaves. (a) Standard sample containing all six catechins; (b) laboratory sample. GC, gallocatechin; EGC, epigallocatechin; C, catechin; EC, epicatechin; EGCG, epigallocatechin gallate; ECG, epicatechin gallate.

and its gallolyl derivatives EGC, ECG, and EGCG) constitute approximately $90 \%$ of the TCs in tea leaves. ${ }^{41}$ In this study, the TC concentration increased by $2.29 \mathrm{mg} \mathrm{g}^{-1}$ in tea plants harvested in autumn (Table 3). Nagata and Sakai ${ }^{42}$ reported that the order of catechin concentrations in tea leaves (C. sinensis var. sinensis) was EGCG> EGC $>$ ECG from highest to lowest. In Assam tea (C. sinensis var. assamica) leaves, the order was EGCG $>E C G>E C$. These different results indicate that the composition of each catechin substance varies in

Table 3. Concentrations of catechin in tea bud leaves during spring and autumn

\begin{tabular}{|c|c|c|c|c|c|c|c|c|c|c|}
\hline \multirow[b]{3}{*}{ Variety } & \multicolumn{5}{|c|}{ Spring } & \multicolumn{5}{|c|}{ Autumn } \\
\hline & \multicolumn{2}{|c|}{ Non-esterified catechins } & \multicolumn{2}{|c|}{ Esterified catechins } & \multirow[b]{2}{*}{$\mathrm{TC} / \mathrm{mg} \mathrm{g}^{-1}$} & \multicolumn{2}{|c|}{ Non-esterified catechins } & \multicolumn{2}{|c|}{ Esterified catechins } & \multirow[b]{2}{*}{$\mathrm{TC} / \mathrm{mg} \mathrm{g}^{-1}$} \\
\hline & Content/mg g ${ }^{-1}$ & Ratio/\% & Content/mg g $\mathrm{g}^{-1}$ & Ratio/\% & & Content/mg g ${ }^{-1}$ & Ratio/\% & Content/mg g ${ }^{-1}$ & Ratio/\% & \\
\hline AJBC & $1.86 \pm 0.24$ & 38.8 & $2.95 \pm 0.43$ & 61.2 & $4.82 \pm 0.87$ & $4.96 \pm 0.68$ & 0.6 & $3.37 \pm 0.86$ & 0.4 & $8.33 \pm 1.54$ \\
\hline LJCY & $2.18 \pm 0.35$ & 40.0 & $3.28 \pm 0.42$ & 60.0 & $5.46 \pm 0.77$ & $2.75 \pm 0.43$ & 0.42 & $3.72 \pm 0.80$ & 0.58 & $6.47 \pm 1.23$ \\
\hline WNZ & $2.02 \pm 0.15$ & 42.2 & $2.77 \pm 0.37$ & 57.8 & $4.79 \pm 0.52$ & $4.54 \pm 0.47$ & 0.55 & $3.65 \pm 0.87$ & 0.45 & $8.19 \pm 1.34$ \\
\hline FDDH & $2.35 \pm 0.27$ & 36.5 & $4.09 \pm 0.46$ & 63.5 & $6.44 \pm 0.73$ & $4.75 \pm 0.62$ & 0.53 & $4.27 \pm 1.52$ & 0.47 & $9.02 \pm 2.14$ \\
\hline FDDB & $2.26 \pm 0.17$ & 37.5 & $3.78 \pm 0.38$ & 62.5 & $6.04 \pm 0.55$ & $5.44 \pm 0.86$ & 0.6 & $3.61 \pm 1.55$ & 0.4 & $9.05 \pm 2.41$ \\
\hline LJ43 & $2.01 \pm 0.13$ & 38.1 & $3.27 \pm 0.59$ & 61.9 & $5.28 \pm 0.72$ & $3.04 \pm 0.53$ & 0.42 & $4.24 \pm 0.91$ & 0.58 & $7.27 \pm 1.44$ \\
\hline ZN117 & $2.25 \pm 0.24$ & 38.9 & $3.54 \pm 0.44$ & 61.1 & $5.79 \pm 0.68$ & $4.05 \pm 0.48$ & 0.53 & $3.65 \pm 0.83$ & 0.47 & $7.70 \pm 1.31$ \\
\hline PYTZ & $2.42 \pm 0.36$ & 44.7 & $3.00 \pm 0.46$ & 55.3 & $5.42 \pm 0.82$ & $3.09 \pm 0.51$ & 0.48 & $3.31 \pm 1.01$ & 0.52 & $6.40 \pm 1.52$ \\
\hline
\end{tabular}

The mean \pm SD of three biological replicates are presented.

Ratio is the proportion of the content of ECs or NECs and TC; non-esterified catechins including C, EC, EGC, and GC; esterified catechins including EGCG and ECG; TC, total catechins. 

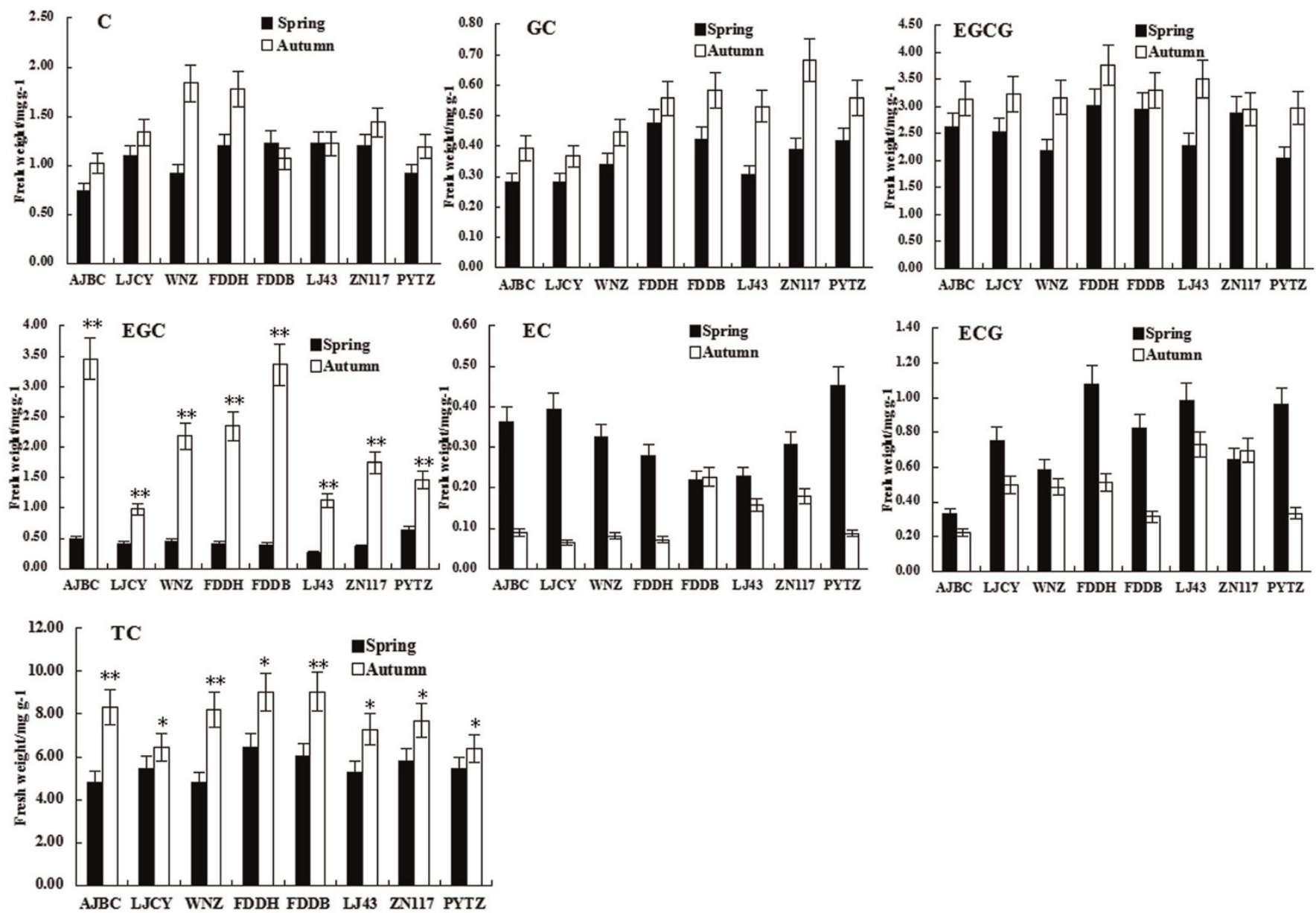

Figure 3. Catechin accumulations of bud leaves in eight cultivars of tea plants harvested in spring and autumn. Error bars indicate SE of three biological replicates. Asterisks indicate that the content was significantly different $(* P \leqslant 0.05, * * P \leqslant 0.01)$.

different cultivars. EGC concentrations in the eight cultivars of tea plants harvested in autumn in this study were significantly higher than in those harvested in spring (Figure 3). Thus, we hypothesized that the higher EGC and EGCG concentrations in autumn tea might be the central reason for its higher catechin levels $(P<0.01)$. EGC, an epimer of EGCG at the C-2 position, is easily detected in tea infusions. In our research, tea harvested in autumn contains higher EGC concentrations (Figure 3). Sano et al. ${ }^{43}$ reported that EGC is produced from the epimerization of EGCG via heat treatment. This finding may explain why the EGC concentrations were higher in autumn tea than in spring tea. In addition, catechins are classified as dihydroxylated catechins or trihydroxylated catechins based on the number of hydroxyls in the B-ring. The ratio of dihydroxylated to trihydroxylated catechin ((EC+ ECG):(EGC+EGCG + GC)) and the level of TC can be used as indicators of better tea quality. ${ }^{40}$ The higher the ratio, the better the tea quality. In this research, the ratio decreased gradually and ranged from 0.10 to
0.37 in autumn tea. Ortho-dihydroxy B-ring-substituted flavonoids may inhibit the generation of free radicals through the chelation of metal ions. ${ }^{27,44,45}$ These unique properties of flavonoids with a catechin group in the B-ring may explain why the ratio of dihydroxy to trihydroxy B-ring-substituted catechins decreased in autumn tea. Based on Table 4, we concluded that season of tea harvest affects catechin accumulation in tea plants. These results indicate improved tea catechin quality in teas harvested in spring. In addition, the quality of taste of the tea harvested in spring was better than of that harvested in autumn, probably owing to the higher EGC content in autumn tea.

\section{The relationship between gene expression and catechin} accumulation

Tea is an important commercial crop known for its flavonoid compounds, such as catechins, which are important in beverages and medicinal use. We found that cultivar, season, and cultivar $\times$ season

Table 4. The effect of cultivar, season, and cultivar $\times$ season on the accumulation of catechin in tea plants.

\begin{tabular}{|c|c|c|c|c|c|}
\hline Source & DF & Sum of squares & Mean square & F-Value & $\operatorname{Pr}>F$ \\
\hline Cultivar & 7 & 20.09196262 & 2.87028037 & 46.70 & $<0.0001^{* *}$ \\
\hline Season & 1 & 63.62509015 & 63.62509015 & 1035.25 & $<0.0001 * *$ \\
\hline Cultivar $\times$ season & 7 & 5.81875893 & 0.83125128 & 13.53 & $<0.0001 * *$ \\
\hline Error & 32 & 1.96666667 & 0.06145833 & & \\
\hline Corrected total & 47 & 91.50247837 & & & \\
\hline
\end{tabular}

Asterisks indicate that the $D$-value was significantly different $(* * P \leqslant 0.01)$. 
Table 5. Correlation analysis between the accumulation of catechin and biosynthesis-related gene expression in spring. $\beta$-actin was used as an internal control

\begin{tabular}{|c|c|c|c|c|c|c|c|}
\hline & $\mathrm{C}$ & EC & GC & EGC & ECG & EGCG & $\mathrm{TC}$ \\
\hline$P A L$ & -0.114 & -0.065 & 0.649 & 0.071 & $0.757^{*}$ & 0.581 & $0.773^{*}$ \\
\hline $\mathrm{C} 4 \mathrm{H}$ & -0.643 & 0.622 & 0.346 & 0.482 & -0.416 & -0.553 & 0.544 \\
\hline $\mathrm{CHS}$ & $-0.684^{*}$ & 0.126 & 0.259 & 0.381 & -0.534 & -0.419 & -0.406 \\
\hline $\mathrm{CHI}$ & -0.519 & 0.552 & 0.242 & -0.519 & -0.633 & 0.552 & 0.509 \\
\hline F3H & -0.628 & 0.094 & 0.247 & 0.227 & -0.573 & -0.660 & $-0.689 *$ \\
\hline$F 3^{\prime} H$ & 0.307 & -0.385 & 0.596 & 0.388 & 0.371 & $0.899 * *$ & 0.547 \\
\hline$F 3^{\prime} 5^{\prime} H$ & -0.331 & 0.035 & -0.275 & -0.214 & -0.476 & $-0.774^{*}$ & $-0.727^{*}$ \\
\hline DFR & $0.727^{*}$ & 0.146 & $0.720 *$ & 0.342 & 0.646 & $0.913 * *$ & $0.920 * *$ \\
\hline ANS & $0.831^{*}$ & $-0.764 *$ & 0.399 & $-0.732 *$ & $0.889 * *$ & 0.251 & $-0.830 *$ \\
\hline$\angle A R$ & $-0.895 * *$ & $0.722 *$ & -0.393 & 0.643 & 0.541 & -0.288 & $0.707 *$ \\
\hline$A N R$ & $0.899 * *$ & -0.620 & $0.744 *$ & -0.564 & $0.730 *$ & 0.382 & 0.453 \\
\hline UFGT & 0.536 & $-0.707 *$ & -0.207 & $-0.733^{*}$ & 0.327 & 0.334 & -0.126 \\
\hline
\end{tabular}

Asterisks indicate that the gene was significant correlated with the catechin content $(* P \leqslant 0.05, * * P \leqslant 0.01)$.

exert significant effects on catechin concentrations in tea plants (Table 4). The results indicate that the $P A L, F 3^{\prime} 5^{\prime} H$, and DFR genes, which are involved in the catechin biosynthesis pathway, are positively correlated with catechin concentrations in tea bud leaves harvested both in spring and autumn. $\mathrm{FHH}$ and ANS expression decreased in autumn. These results indicate that the season plays a very important role in regulating the expression of genes related to catechin biosynthesis, such as F3H and ANS.

Flavan-3-ols (also known as catechins) are mainly produced via the naringenin-chalcone $\rightarrow$ naringenin $\rightarrow$ dihydrokaempferol pathway. ${ }^{11}$ Eungwanichayapant and Popluechai ${ }^{30}$ reported that increased tea catechin concentrations are attributed to the increased expression of genes involved in catechin biosynthesis. As shown in Figure 1, PAL catalyzes the deamination of phenylalanine to produce trans-cinnamic acid, which is converted to p-coumaric acid via an oxidative reaction catalyzed by the cytochrome P450 enzyme $\mathrm{C} 4 \mathrm{H}$. Research indicates that the $\mathrm{C} 4 \mathrm{H}$ gene is involved in the catechin pathway and that its expression is associated with catechin accumulation. ${ }^{14,46} \mathrm{~F}^{\prime} \mathrm{H}$, $F 3^{\prime} 5^{\prime} H$, and DFR catalyze the reduction of flavanones to leucoanthocyanidin. ${ }^{12,15-18} \mathrm{C}$ and EC are hydroxylated by the $\mathrm{F}^{\prime} \mathrm{S}^{\prime} \mathrm{H}$ gene. ${ }^{47-49}$ $F 3^{\prime} 5^{\prime} H$ exhibits a $3^{\prime} 4^{\prime} 5^{\prime}$-hydroxylation pattern, such as GC, EGC, and EGCG. In addition, the $3^{\prime}, 4^{\prime}$-dihydroxylation of the B-ring, as is evident in quercetin, substantially increases the antioxidant activity of flavonoids as compared with B-ring monohydroxylated flavonols. DFR is a key enzyme in the catechin flavonoid pathway and catalyzes important steps in the control of metabolic fluxes that feed into biosynthetic pathway branches, thus producing anthocyanins and proanthocyanidins. ${ }^{19}$ DFR is overexpressed in calluses harvested in sufficient light ${ }^{50}$ and bud leaves ${ }^{51,52}$ but downregulated in leaves grown in the absence of light. $^{24}$ Therefore, tea catechin biosynthesis is critically dependent upon the products of these enzymes. ${ }^{46}$ Our research demonstrated that $P A L, F 3^{\prime} 5^{\prime} H$, and DFR exhibited the same positive correlation with TC concentrations in tea bud leaves harvested in spring and autumn (Tables 5 and 6). This result indicates that these three genes may serve as core factors in the control of catechin biosynthesis in tea plants regardless of the harvest season.

$L A R$ is the only enzyme that has been found to catalyze the conversion of leucocyanidin to $C$ in many plants. ${ }^{53} \mathrm{~A}$ negative correlation between $C$ content and $L A R$ expression level was also observed in the bud leaves of the eight different tea varieties harvested, both in spring and autumn (Tables 5 and 6 ). We also found that $L A R$ transcripts decreased and $C$ and GC concentrations increased in autumn tea (Figure 3).

TC concentrations in autumn tea were significantly higher than in spring tea given the greater EGC content in the eight tea plant cultivars harvested in autumn (Figure 3). High EGC concentrations in green tea have been reported by Eungwanichayapant and Popluechai. ${ }^{30}$ Based on the correlation analysis of catechin levels and the expression of genes involved in catechin biosynthesis, we observed positive correlations between PAL, C4H, F3H, and DFR expression and EGC accumulation in tea plants harvested in autumn, with correlation coefficients of $0.710,0.763,0.884$, and 0.707 (Table 6), respectively; only ANS was negatively correlated with EGC concentrations in tea plants harvested in spring (Table 5).

$\mathrm{F} 3 \mathrm{H}$ is an essential gene in the catechin biosynthetic pathway and catalyzes the stereo-specific hydroxylation of (2S)-naringenin and (2S)-eriodictyol to form (2R,3R)-dihydrokaempferol and (2R,3R)dihydroquercetin, respectively. ${ }^{54} \mathrm{~F} 3 \mathrm{H}$ regulates the types and

Table 6. Correlation analysis between the accumulation of catechin and biosynthesis-related gene expression in autumn. $\beta$-actin was used as an internal control

\begin{tabular}{|c|c|c|c|c|c|c|c|}
\hline & C & $\mathrm{EC}$ & $\mathrm{GC}$ & EGC & ECG & EGCG & $\mathrm{TC}$ \\
\hline$P A L$ & -0.261 & 0.441 & 0.379 & $0.710 *$ & -0.751 & $0.736 *$ & $0.713^{*}$ \\
\hline $\mathrm{C} 4 \mathrm{H}$ & -0.481 & -0.024 & -0.324 & $0.763 *$ & $-0.961 * *$ & -0.258 & $0.745^{*}$ \\
\hline $\mathrm{CHS}$ & 0.464 & -0.569 & 0.143 & -0.261 & 0.247 & 0.167 & -0.014 \\
\hline $\mathrm{CHI}$ & 0.113 & -0.381 & $-0.707^{*}$ & 0.515 & $0.707 *$ & 0.179 & -0.106 \\
\hline $\mathrm{F} 3 \mathrm{H}$ & 0.577 & -0.188 & 0.216 & $0.884 * *$ & $-0.971 * *$ & -0.267 & $0.707 *$ \\
\hline$F 3^{\prime} H$ & 0.039 & -0.310 & $-0.737 *$ & 0.091 & $0.707 *$ & -0.547 & -0.213 \\
\hline$F 3^{\prime} 5^{\prime} H$ & 0.527 & -0.148 & -0.451 & 0.641 & $-0.713^{*}$ & -0.040 & $-0.781 *$ \\
\hline$D F R$ & 0.372 & $0.744 *$ & 0.179 & $0.707^{*}$ & -0.335 & $0.795 *$ & $0.728 *$ \\
\hline ANS & $0.732 *$ & -0.241 & $0.742 *$ & 0.384 & 0.487 & 0.556 & $0.709 *$ \\
\hline$L A R$ & $-0.721^{*}$ & $0.721 *$ & 0.073 & 0.501 & -0.466 & -0.314 & 0.314 \\
\hline$A N R$ & -0.100 & 0.444 & $0.884 * *$ & 0.112 & $0.707 *$ & 0.079 & 0.120 \\
\hline UFGT & $-0.707^{*}$ & -0.232 & -0.412 & -0.235 & -0.351 & -0.519 & 0.562 \\
\hline
\end{tabular}

Asterisks indicate that the gene was significant correlated with the catechin content $(* P \leqslant 0.05, * * P \leqslant 0.01)$. 

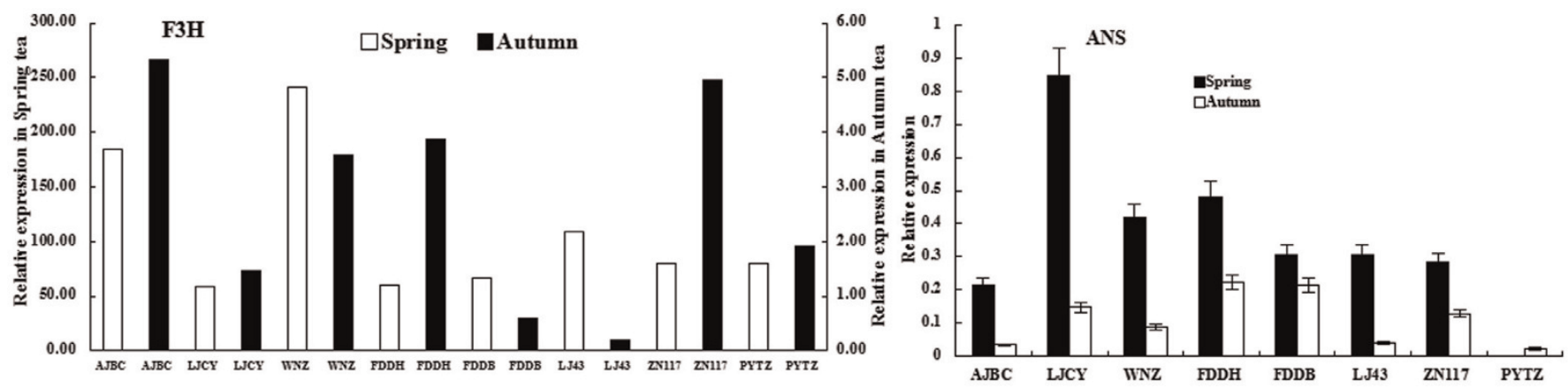

Figure 4. Expression of genes $F 3 H$ and ANS encoding catechin biosynthesis enzymes in bud leaves of eight varieties of tea plants relative to $\beta$ actin gene, used as an internal control. Error bars indicate SD of three technical replicates.

quantities of flavonoids and also plays an important role in resistance to biotic and abiotic stresses. ${ }^{55} \mathrm{~F} 3 \mathrm{H}$ expression is controlled and regulated by catechin levels. ${ }^{49,56}$ In the present study, a positive correlation between EGC and TC concentrations and F3H expression was noted in the bud leaves of eight cultivars harvested in autumn, whereas a negative correlation was evident in spring. Zhang et al. $^{57}$ demonstrated that $\mathrm{F} 3 \mathrm{H}$ expression was increased in Reaumuria trigyna under drought as well as cold stresses. The materials were harvested in northern subtropical climate zones; a notable feature of these areas is the monsoon. The amount of sunshine and precipitation in winter and spring seasons is less than that in the summer and autumn seasons. These climatic characteristics potentially explain why $\mathrm{F} 3 \mathrm{H}$ expression was markedly downregulated in the tea bud leaves of eight cultivars harvested in autumn (Figure 4). Thus, EGC may be hydroxylated by $\mathrm{F} 3 \mathrm{H}$, which may serve as a key gene in the control of catechin concentrations in tea plants harvested in different seasons. Therefore, further studies are required to better understand the relationship between $\mathrm{F} 3 \mathrm{H}$ gene expression and catechin accumulation in tea plants harvested in autumn.

The metabolic genes involved in EGC biosynthesis include ANS and ANR. ANS expression results in the accumulation of ECs and is a key enzyme at the branch points of catechin biosynthesis. Through the catalysis of $A N S$ and $A N R$, leucoanthocyanin is transformed into 2,3-trans-flavan-3-ols, such as EC and EGC. ${ }^{11}$ Hong et al. ${ }^{32}$ demonstrated that ANS is the key enzyme at the branch points of catechin biosynthesis, which results in the accumulation of ECs. They also found that darkness reduced ANS expression and EGC accumulation. In our research, a positive correlation was noted between EGC and TC concentrations and ANS expression in the bud leaves of eight cultivars harvested in autumn, whereas a negative correlation was evident in spring. ANS expression was downregulated in autumn tea as compared with spring tea, and higher EGC concentrations were observed (Figures 3 and 4). In contrast, EC and ECG concentrations were lower in autumn tea (Figure 3 ). It is a novel finding that different harvest seasons exert different effects on the expression patterns of genes involved in the phenylpropanoid pathway. This finding supports the hypothesis that ANS may be a critical gene involved in catechin accumulation. The facilitation of EGC production necessitates the adjustment of EC and ECG biosynthesis in the opposite direction. This finding demonstrates how tea plants maintain the balance of phenylpropanoid metabolism in response to environmental cues. Studies in which the temperature and light duration are varied would be required to assess the exact function of ANS in tea plants harvested in autumn.

\section{CONCLUSIONS}

In summary, this study was the first to examine the expression of most of the catechin biosynthesis pathway genes (with the exception of FGS) and measure the changes in catechin concentrations in the leaves of eight tea plant cultivars harvested in spring and autumn. Our results explain the basic causes of the increased astringency of autumn tea. These results suggest that $\mathrm{FH}$ and ANS are the most important genes involved in the catechin biosynthetic pathway in tea plants. In addition, negative correlations between F3H and ANS expression and catechin levels were identified in spring tea, whereas a positive correlation was observed in autumn tea. We hypothesize that the suppression of ANS and $\mathrm{F} 3 \mathrm{H}$ expression potentially alters catechin biosynthesis in tea plants during spring and autumn. Catechin accumulation was markedly increased in autumn tea.

\section{CONFLICT OF INTEREST}

The authors declare no conflict of interest.

\section{ACKNOWLEDGMENTS}

We thank the experimental tea garden of the Tea Research Institute of Jiangsu Province for generous access to its tea leaves. This research was financially supported by the National Program on Key Basic Research Projects (The 973 Program: 2012CB113900) and Jiangsu Key Laboratory for Horticultural Crop Genetic Improvement.

\section{REFERENCES}

1 Fang WP, Meinhardt LW, Tan HW et al. Varietal identification of tea (Camellia sinensis) using nanofluidic array of single nucleotide polymorphism (SNP) markers. Hortic Res 2014; 1: 1-8.

2 Friedman M, Mackey BE, Kim HJ et al. Structure-activity relationships of tea compounds against human cancer cells. J Agric Food Chem 2007; 55: 243-253.

3 Higdon JV, Frei B. Tea catechins and polyphenols: health effects, metabolism, and antioxidant functions. Crit Rev Food Sci Nutr 2003; 43: 89-143.

4 Ho CT, Lin JK, Shahidi F. Tea and Tea Products: Chemistry and Health-promoting Properties. Boca Raton, FL: CRC Press; 2008. p320.

5 Miura Y, Chiba T, Tomita I et al. Green tea polyphenols (flavan 3-ols) prevent oxidative modification of low density lipoproteins: an ex vivo study in humans. J Nutr Biochem 2001; 11: 216-222.

6 Wang YC, Bachrach U. The specific anti-cancer activity of green tea (-)-epigallocatechin-3-gallate (EGCG). Amino Acids 2002; 22: 131-143.

7 Graham HN. Green tea composition, consumption, and polyphenol chemistry. Prev Med 1992; 21: 334-350.

8 Shirley BW. Flavonoid biosynthesis: a colorful model for genetics, biochemistry, cell biology, and biotechnology. Plant Physiol 2001; 126: 485-493.

9 Wang YS, Gao LP, Shan Y et al. Influence of shade on flavonoid biosynthesis in tea (Camellia sinensis (L.) O.Kuntze). Sci Hort 2012; 141: 7-16.

10 Xie DY, Sharma SB, Nancy LP et al. Role of anthocyanidin reductase, encoded by BANYULS in plant flavonoid biosynthesis. Science 2003; 299: 396-399.

11 Ashihara H, Deng WW, Mullen W et al. Distribution and biosynthesis of flavan-3-ols in Camellia sinensis seedlings and expression of genes encoding biosynthetic enzymes. Phytochemistry 2010; 71: 559-566.

12 Forkmann G, Heller W, Grisebach H. Anthocyanin biosynthesis in flowers of matthiola incana. Flavonone 3-and flavonoid 30-hydroxylases. Z Naturforsch 1980; p35. 
13 Moustafa E, Wong E. Purification and properties of chalcone-flavanone isomerase from soya bean seed. Phytochemistry 1967; 6: 625-632.

14 Rani A, Singh K, Sood P et al. p-Coumarate: CoA ligase as a key gene in the yield of catechins in tea (Camellia sinensis (L.) O. Kuntze). Func Integr Genomics 2009; 9: 271275.

15 Reddy AR, Britsch L, Salamini F et al. The a1 (anthocyanin-1) locus in Zea mays encodes dihydroquercetin reductase. Plant Sci 1987; 52: 7-13.

16 Reddy BS, Maruyama H, Kelloff G. Dose-related inhibition of colon carcinogenesis by dietary piroxicam, a nonsteroidal antiinflammatory drug, during different stages of rat colon tumor development. Cancer Res 1987; 47: 5340-5346.

17 Toda K, Yang D, Yamanaka $\mathrm{N}$ et al. A single-base deletion in soybean flavonoid 3'hydroxylase gene is associated with gray pubescence color. Plant Mol Biol 2002; 50: 187-196.

18 Toda M, Tulic MK, Levitt RC et al. A calcium-activated chloride channel (hclca1) is strongly related to il-9 expression and mucus production in bronchial epithelium of patients with asthma. J Allergy Clin Immunol 2002; 109: 246-250.

19 Xie DY, Jackson LA, Cooper JD et al. Molecular and biochemical analysis of two cDNA clones encoding dihydroflavonol-4-reductase from Medicago truncatula. Plant Physiol 2004; 134: 979-994.

20 Fendri A, Masmoudi A, Khabir A et al. Inactivation of rassf1a, rarbeta2 and dapkinase by promoter methylation correlates with lymph node metastasis in nasopharyngeal carcinoma. Cancer Biol Ther 2009; 8: 444-451.

21 Harborne JB, Grayer RJ. Flavonoids and Insects. In: Harborne JB, editor. flavonoids: advances in research since 1986. UK, London; Chapman \& Hall; 1994; 589-618.

22 Pang Y, Abeysinghe ISB, He J et al. Functional characterization of proanthocyanidin pathway enzymes from tea and their application for metabolic engineering. Plant Physiol 2013; 161: 1103-1116.

23 Winkel-Shirley. Flavonoid biosynthesis: a colorful model for genetics, biochemistry, cell biology, and biotechnology. Plant Physiol 2001; 126: 485-493.

24 Wang YS, Gao LP, Wang ZR et al. Light-induced expression of genes involved in phenylpropanoid biosynthetic pathways in callus of tea (Camellia sinensis (L.) $\mathrm{O}$ Kuntze). Sci Hort 2012; 133: 72-83.

25 Takeuchi A, Matsumoto S, Hayatsu M. Effects of shading treatment on the expression of the genes for chalcone synthase and phenylalanine ammonialyase in tea plant (Camellia, sinensi). Bull Natl Res Inst Veg Ornam Plants \& Tea, Japan (B) 1995; 8: 1-9.

26 Winkel-Shirley B. Biosynthesis of flavonoids and effects of stress. Curr Opin Plant Biol 2002; 5: 218-223.

27 Agati G, Tattini M. Multiple functional roles of flavonoids in photo protection. New Phytol 2010; 186: 786-793.

28 Lillo C, Lea U, Ruoff P. Nutrient depletion as a key factor for manipulating gene expression and product formation in different branches of the flavonoid pathway. Plant Cell Environ 2007; 31: 587-601.

29 Hipskind J, Wood K, Nicholson RL. Localized stimulation of anthocyanin accumulation and delineation of pathogen ingress in maize genetically resistant to Bipolaris maydis race O. Physiol Mol Plant Pathol 1996; 49: 247-256.

30 Eungwanichayapant PD, Popluechai S. Accumulation of catechins in tea in relation to accumulation of mRNA from genes involved in catechin biosynthesis. Plant Physiol Biochem 2009; 47: 94-97.

31 Xiong LG, Lia J, Lia YH et al. Dynamic changes in catechin levels and catechin biosynthesis-related gene expression in albino tea plants (Camellia sinensis L.) Plant Physiol Biochem 2013; 71: 132-143.

32 Hong GJ, Wang J, Zhang $Y$ et al. Biosynthesis of catechin components is differentially regulated in dark-treated tea (Camellia sinensis L.). Plant Physiol Biochem 2014; 78: 49-52.

33 Zhang XB. Differences of polyphenols content in Anxi TieGuanYin tea among different seasons and relationship between polyphenols and tea quality. Agric Sci Technol 2014; 15: 1191-1195.

34 Peng P, Xie QZ, Li PW et al. Studies on the allelopathy components of tea. Southwest China J Agric Sci 2009; 1: 67-70. Chinese.

35 Wei J, Tian YH, Liang YF et al. Effect of produce autotoxic-chemical of tea tree on environment factor. Chinese Agric Sci Bull 2003; 19: 53-56. Chinese.

36 Gulati A, Rawat R, Singh B et al. Application of microwave energy in the manufacture of enhanced-quality green tea. J Agric Food Chem 2004; 51: 4764 4768
37 Li DH, Martini N, Wu ZM et al. Development of an isocratic HPLC method for catechin quantification and its application to formulation studies. Fitoterapia 2012; 83: 1267-1274.

38 Zhang RR, Wang SY, Wang FJ et al. Simultaneous determination of eight catechins in Chinese tea by high performance liquid chromatography. Heilongjiang Med $J$ 2011; 5: 681-683. Chinese.

39 Livak KJ, Schmitten TD. Analysis of relative gene expression data using real-time quantitative PCR and the $2^{-\Delta \Delta C}$ T method. Methods 2001; 25: 402-408

40 Wei K, Wang L, Zhou J et al. Catechin contents in tea (Camellia sinensis) as affected by cultivar and environment and their relation to chlorophyll contents. Food Chem 2011; 125: 44-48.

41 Zhen YS, Ed. Tea: Bioactivity and Therapeutic Potential. Boca Raton, FL: CRC Press; 2004. p280.

42 Nagata T, Sakai S. Differences in caffeine, flavonols and amino acids contents in leaves of cultivated species of Camellia. Jpn J Breed 1984; 34: 459-467.

43 Sano M, Tabata M, Suzuki M et al. Simultaneous determination of twelve tea catechins by high-performance liquid chromatography with electrochemical detection. Analyst 2001; 126: 816-820.

44 Brown JE, Khodr H, Hider RC et al. Structural dependence of flavonoid interactions with $\mathrm{Cu}^{2+}$ ions: implications for their antioxidant properties. Biochem J 1998; 330: 1173-1178.

45 Smyk B, Pliszka B, Drabent R. Interaction between Cyanidin 3-glucoside and Cu (II) ions. Food Chem 2008; 107: 1616-1622.

46 Singh K, Kumar S, Rani A et al. Phenylalanine ammonia-lyase(PAL) and cinnamate 4-hydroxylase $(\mathrm{C} 4 \mathrm{H})$ and catechins (flavan-3-ols) accumulation in tea. Funct Integr Genomics 2009; 9: 125-134.

47 Harborne JB. The Flavonoids: Advances in Research since 1980. New York: Chapman \& Hall; 1988. p621. Softcover reprint of the original 1st ed.

48 Holton TA, Cornish EC. Genetics and biochemistry of anthocyanin biosynthesis. Plant Cell 1995; 7: 1071-1083.

49 Punyasiri PA, Abeysinghe IS, Kumar V et al. Flavonoid biosynthesis in the tea plant Camellia sinensis: properties of enzymes of the prominent epicatechin and catechin pathways. Arch Biochem Biophys 2004; 431: 22-30.

50 Yang DQ, Liu YJ, Sun ML et al. Differential gene expression in tea (Camellia sinensis L.) calli with different morphologies and catechin contents. J Plant Physiol 2012; 169: 163-175.

51 Mamati GE, Liang Y, Lu J. Expression of basic genes involved in tea polyphenol synthesis in relation to accumulation of catechins and total tea polyphenols. J Sci Food Agric 2005; 86: 459-464.

52 Nakajima J, Tanaka Y, Yamazaki $M$ et al. Reaction mechanism from leucoanthocyanidin to anthocyanidin 3-glucoside, a key reaction for coloring in anthocyanin biosynthesis. J Biol Chem 2001; 276: 25797-25803.

53 Abrahams S, Tanner GJ, Larkin PJ et al. Identification and biochemical characterization of mutants in the proanthocyanidin pathway in Arabidopsis. Plant Physiol 2002; 130: 561-576.

54 Britsch L, Grisebach H. Purification and characterization of (2S)-flavanone 3hydroxylase from Petunia hybrida. Eur J Biochem 1986; 156: 569-577.

55 Jiang F, Wang JY, Jia HF et al. RNAi-mediated silencing of the flavanone 3Hydroxylase gene and its effect on flavonoid biosynthesis in strawberry fruit. J Plant Growth Regul 2013; 32: 182-190.

56 Singh K, Rani A, Kumar S et al. An early gene of the flavonoid pathway, flavanone3hydroxylase, exhibits a positive relationship with the concentration of catechins in tea (Camellia sinensis). Tree Physiol 2008; 28: 1349-1356.

57 Zhang HR, Zhao LX, Wang J et al. Cloning and functional analysis of two flavanone3-hydroxylase genes from Reaumuria trigyna. Acta Physiol Plant 2014; 36: 1221 1229.

(i) (2) This work is licensed under a Creative Commons Attribution-

cc. NonCommercial-ShareAlike 3.0 Unported License. The images or other third party material in this article are included in the article's Creative Commons license, unless indicated otherwise in the credit line; if the material is not included under the Creative Commons license, users will need to obtain permission from the license holder to reproduce the material. To view a copy of this license, visit http://creativecommons.org/ licenses/by-nc-sa/3.0/ 